\title{
Real-time monitoring of the silicidation process of tungsten filaments at high temperature used as catalysers for silane decomposition
}

\author{
$\underline{\text { O. Nos }^{1}}$, P.A. Frigeri ${ }_{2}^{1}$ J. Bertomeu ${ }^{1}$. \\ ${ }^{1}$ Dept. de Física Aplicada i Òptica, Universitat de Barcelona \\ Av. Diagonal 645, 08028-Barcelona, Spain, $\mathrm{Ph}:+34934039221$, fax: +34934039219 \\ E-mail of corresponding author: oriol.nos@gmail.com
}

\begin{abstract}
The scope of this work is the systematic study of the silicidation process affecting tungsten filaments at high temperature $\left(1900^{\circ} \mathrm{C}\right)$ used for silane decomposition in the hot-wire chemical vapour deposition technique (HWCVD). The correlation between the electrical resistance evolution of the filaments, $R_{\mathrm{fil}}(t)$, and the different stages of the their silicidation process is exposed. Said stages correspond to: the rapid formation of two $\mathrm{WSi}_{2}$ fronts at the cold ends of the filaments and their further propagation towards the middle of the filaments; and, regarding the hot central portion of the filaments: a initial stage of silicon dissolution into the tungsten bulk, with a random duration for asmanufactured filaments, followed by the inhomogeneous nucleation of $\mathrm{W}_{5} \mathrm{Si}_{3}$ (which is later replaced by $\mathrm{WSi}_{2}$ ) and its further growth towards the filaments core. An electrical model is used to obtain real-time information about the current status of the filaments silicidation process by simply monitoring their $R_{\text {fil }}(t)$ evolution during the HWCVD process. It is shown that implementing an annealing pre-treatment to the filaments leads to a clearly repetitive trend in the monitored $R_{\text {fil }}(t)$ signatures. The influence of hydrogen dilution of silane on the filaments silicidation process is also discussed.
\end{abstract}

Keywords:tungsten silicides, tungsten filament, hot-wire chemical vapour deposition, HWCVD, Cat-CVD. 


\section{Introduction}

The properties of thin film tungsten (W) silicides have been widely studied in the last decades for their application as contact materials in microelectronics or as hightemperature protective coatings for $\mathrm{W}$ and $\mathrm{W}$ alloys used in oxidative atmospheres [14]. In this context, some works dealing with the growth process of silicides $\left(\mathrm{WSi}_{2}\right.$ and $\mathrm{W}_{5} \mathrm{Si}_{3}$ ) in $\mathrm{W}$ metal films have been published in the last years [5-7]. The study of silicides formation processes in $\mathrm{W}$ filaments has also attracted much attention from the scientific community in the context of the hot-wire chemical vapour deposition (HWCVD) of silicon ( $\mathrm{Si}$ ) related materials [8-13]. In this CVD technique, W filaments are usually used to catalytically decompose the molecules of a raw gas, like for instance silane $\left(\mathrm{SiH}_{4}\right)$, and generate the silicon film precursors. It is well known that the silicidation processes affecting such $\mathrm{W}$ catalytic filaments cause their premature breakage thus compromising the stability of the HWCVD process. However, in these previous studies there are no data or very little explanation regarding the silicides formation process in HWCVD for filament temperatures higher than $\mathrm{T}_{\text {fil }}>1800^{\circ} \mathrm{C}$. This filament temperature range is very interesting because it allows achieving very high deposition rates for either amorphous (a-Si:H) or microcrystalline ( $\mu \mathrm{c}-\mathrm{Si}: \mathrm{H})$ silicon $[14,15]$ with a reduced silicides growth rate $[9,12]$.

In this paper we will present a systematic study of the silicidation process of $\mathrm{W}$ filaments at $T_{\text {fil }}=1900^{\circ} \mathrm{C}$ exposed to a pure silane $\left(\mathrm{SiH}_{4}\right)$ atmosphere at a partial pressure of $2 \mathrm{~Pa}$. Several silicidation tests have been performed varying the exposure time to the $\left(\mathrm{SiH}_{4}\right)$ atmosphere. The evolution of the electric resistance, $R_{\mathrm{fil}}(t)$, of the tested filaments was monitored and the physicochemical status of said filaments was checked $e x$-situ by means of scanning electron microscopy (SEM), energy-dispersive xray spectroscopy (EDX) and Raman spectroscopy.

We will show that the silicidation process at the so called "cold ends" of the filaments, i.e. those low-temperature regions close to the electrical contacts, involves the rapid formation of two $\mathrm{WSi}_{2}$ fronts and their further propagation towards the hot central portion of filament. Regarding the hot central portion of the filament, we will show that at these particular $T_{\text {fil }}$, the silicidation process goes through three different stages: an initial stage of Si atoms dissolution in the pure $\mathrm{W}$ bulk without any silicide precipitation; next, the inhomogeneous precipitation of a $\mathrm{W}_{5} \mathrm{Si}_{3}$ silicide layer at the filament surface, nucleating after a period of time which varies randomly; after that, the rapid formation of a $\mathrm{W}_{5} \mathrm{Si}_{3}$ outer corona and its further growth towards the filament core; and finally, the substitution of the previously formed $\mathrm{W}_{5} \mathrm{Si}_{3}$ outer corona by $\mathrm{WSi}_{2}$ once the superficial precipitation of $\mathrm{WSi}_{2}$ takes place. SEM, EDX and Raman analyses of samples taken from the tested filaments have been performed to describe and study such processes. The influence of an annealing pre-treatment of the filaments on their silicidation process will be also exposed. Said annealing treatment leads to a much less random silicides nucleation process, resulting in a repetitive evolution of $R_{\mathrm{fil}}(t)$ for the different tested filaments. 
An electric resistance model, which correlates the evolution of the physicochemical

status of the filaments with their electric resistance evolution $R_{\mathrm{fil}}(t)$ has been built from the comparison of the results obtained from the SEM, EDX and Raman analyses of the tested filaments and their respective $R_{\mathrm{fil}}(t)$ curves. Said model is used to explain the nucleation and growth processes of silicides occurring at the filament cold ends and at the hot central portion of the filaments allowing the monitoring at real time of the filaments silicidation process by simply looking at the evolution of $R_{\mathrm{fil}}(t)$. Finally, it is discussed the effect of adding molecular hydrogen $\left(\mathrm{H}_{2}\right)$ to the silane atmosphere on the silicidation process.

\section{Experimental}

Three different series of silicidation tests were carried out in a single high vacuum chamber connected to silane and hydrogen gas lines. A schematic overview of the experimental setup is shown in Fig. 1. Before starting each test, the chamber was evacuated to less than $10^{-4} \mathrm{~Pa}$ by means of a turbomolecular pump. All silicidation tests were performed using one $\mathrm{W}$ filament of length $L_{\mathrm{fil}}=30 \mathrm{~cm}$ and a diameter of $d_{\mathrm{fil}}=0.125$ $\mathrm{mm}$. Using such thin $\mathrm{W}$ filaments (in comparison with the usual $0.5 \mathrm{~mm}$ diameter filaments used in HWCVD) allows obtaining larger fractions of the initial tungsten material reacted into the different silicide phases for shorter exposure times. In all cases, the filaments were heated up by Joule effect, fixing the electric current to $I=1.63 \mathrm{~A}$ to reach a filament temperature $T_{\mathrm{fil}}=1900^{\circ} \mathrm{C}$. This is the initial filament temperature which was indirectly estimated by solving the Stefan-Boltzmann equation for the given current and using tabulated resistivity and emissivity values for tungsten [16, 17]. By fixing the electric current, it was ensured, as long as the silicides precipitation did not initiate there, that the local temperature at the central portion of the filaments was kept at its initial value of $1900^{\circ} \mathrm{C}$ independently of the on-going silicides formation at the cold ends. The chosen process silane partial pressure of $2 \mathrm{~Pa}$ allows performing accelerated filament silicidation tests, thus making the whole study less time consuming than if the usual deposition conditions for high quality a-Si:H or $\mu \mathrm{c}-\mathrm{Si}: \mathrm{H}$ deposition had been chosen. It is not clear yet, whether the decrease of the process silane partial pressure only decelerates the silicidation process [18-19], or if, below a critical value, the silicidation could be prevented [20].

The resistance evolution $R_{\mathrm{fil}}(t)$ was calculated from the values of the monitored electrical tension applied to the filament. The monitoring always started $(t=0)$ at the moment at which the silane was introduced in the chamber, being the filament already switched on.

In the first series, the $\mathrm{SiH}_{4}$ atmosphere was created by introducing a pure silane flow of $\Phi_{\mathrm{SiH}}=12 \mathrm{sccm}$ into the reactor. This relatively high silane flow ensures that the partial pressure of silane is practically unchanged once the filament is switched on. Different filaments were exposed to the silane atmosphere during a period of time which varied from $3 \mathrm{~min}$ to $190 \mathrm{~min}$. The $\mathrm{WSi}_{2}$ fronts formed at the cold ends of the filaments were analysed ex-situ by means of SEM (Jeol JSM-840) and EDX (INCA Energy 250, 
Oxford Instruments). The propagation velocity of these silicide fronts was measured by direct observation of the shifting of the abrupt decrease in the filament brightness induced by the higher emissivity of $\mathrm{WSi}_{2}$ in comparison to that of pure $\mathrm{W}$ [12]. Cross section samples taken from the central portion of the filaments were also analysed exsitu by SEM and EDX techniques. In addition, Raman signal mapping (Micro Raman T64000JobinYvon, $\lambda_{\text {laser }}=532 \mathrm{~nm}, P_{\text {laser }}=4.3 \mathrm{~mW}$ ) analyses were performed in order to get a composition map of each sample. For this purpose, the characteristic Raman signatures of $\mathrm{W}_{5} \mathrm{Si}_{3}$, showing peaks at 117,170 and $270 \mathrm{~cm}^{-1}$ [7], and of $\mathrm{WSi}_{2}$, with peaks at 335 and $456 \mathrm{~cm}^{-1}[6,7,21]$, were used to identify the formed silicide phases. The electric resistance evolution of all tested filaments was monitored in order to find a relationship between the different trends observed in the corresponding $R_{\mathrm{fil}}(t)$ curves and the results obtained from the SEM, EDX and Raman measurements.

The second series was performed using the same silane atmosphere as the first one but implementing an annealing pre-treatment to the tested filaments (30 min annealing at $1900^{\circ} \mathrm{C}$ in vacuum). The $R_{\mathrm{fil}}(t)$ curves obtained from these samples were clearly repetitive, thus allowing identifying the different stages of the silicidation process affecting the filaments and the construction of an electric model that explained the common trend observed in $R_{\mathrm{fil}}(t)$.

In the third series, it was added to the silane flow $\left(\Phi_{\mathrm{SiH} 4}=12 \mathrm{sccm}\right)$ a flow of molecular hydrogen $\left(\Phi_{\mathrm{H}_{2}}=108 \mathrm{sccm}\right)$. The partial pressure of silane was maintained at $2 \mathrm{~Pa}$ as for the previous series. However, the introduction of molecular hydrogen increased the total process pressure up to $10 \mathrm{~Pa}$. In such a way, it has been possible to check the influence of the hydrogen dilution of the gas mixture in the silicidation process of the filament and the resulting $R_{\text {fil }}(t)$ curve.

The transition regions of the filaments, defined as the portions of filament comprised between the $\mathrm{WSi}_{2}$ silicide fronts propagating from the cold ends and the hot central portion of the filament, were also analysed. The composition map and the length of such transition regions have been determined by optical microscopy imaging and Raman mapping analysis of a longitudinal cross section sample taken from those regions of a tested filament.

\section{Results and discussion}

\section{$3.1 R_{\mathrm{fil}}(t)$ monitoring}

The $R_{\mathrm{fil}}(t)$ evolution of the filaments tested in the first series was monitored to observe any change in $R_{\mathrm{fil}}$ during their silicidation process. Fig. 2 shows the $R_{\mathrm{fil}}(t)$ curves corresponding to the silicidation tests that resulted in samples (cross sections from the central portion of the filaments) wherein it was later identified the presence of silicides $\left(\mathrm{W}_{5} \mathrm{Si}_{3}\right.$ and/or $\left.\mathrm{WSi}_{2}\right)$ by means of EDX and Raman spectroscopy analysis. On the other hand, Fig. 3 shows the $R_{\text {fil }}(t)$ curves corresponding to those silicidation tests wherein the central portion of the filaments did not reveal any presence of silicides formation. 
Looking at Fig. 2 and Fig. 3, it can be seen that the initial tendency of $R_{\mathrm{fil}}(t)$ was quite in Fig. 2 followed a linear tendency with almost identical slopes of $0.06 \pm 0.01 \Omega / \mathrm{min}$. The presence of this linear regime of $R_{\mathrm{fil}}(t)$ is assumed to be linked to the precipitation of the tungsten rich silicide phase, $\mathrm{W}_{5} \mathrm{Si}_{3}$, as it was only observed for those silicidation tests that resulted in samples with presence of silicides formation. For those silicidation tests with a total silane exposure time higher than $60 \mathrm{~min}$, the observed linear tendency was maintained beyond. The only exemptions were the silicidation tests that lasted 140 and $190 \mathrm{~min}$. In these two cases, the $R_{\mathrm{fil}}(t)$ curves abandon such linear tendency at larger exposure times and a dramatic decrease of the electric resistance held afterwards (see inset in Fig. 2). As shown in the next sub-section such trend change in $R_{\mathrm{fil}}(t)$ is probably linked to the precipitation of the $\mathrm{Si}$ rich silicide phase, $\mathrm{WSi}_{2}$, because those silicidation tests were the only ones that resulted in samples with the presence of a $\mathrm{WSi}_{2}$ outer corona

\subsection{SEM, EDX and Raman mapping analysis}

Fig. 4 and Fig. 5 show SEM images of cross section samples taken from the central portion of the filaments tested in the first series and their corresponding Raman signal mappings, wherein green, red and blue colours indicate the presence of pure $\mathrm{W}, \mathrm{W}_{5} \mathrm{Si}_{3}$ and $\mathrm{WSi}_{2}$ respectively. The presence of the different silicide phases was also confirmed by means of EDX measurements. For those silicidation tests with a silane exposure time lower than 30 minutes, (see Fig. 3 to check their corresponding $R_{\mathrm{fil}}(t)$ curves), no presence of silicides was observed in the collected samples, what indicates that Si and $\mathrm{W}$ atoms were forming a solid solution as already suggested by Holt et al. [8]. For these samples, no figures of SEM and Raman are shown here. Otherwise, Figs. 4 and 5 show the results for those samples that correspond to the monitored resistances $R_{\mathrm{fil}}(t)$ showed in Fig. 2, and the one with 30 minutes of exposition showed in Fig. 3. For these samples, the solubility limit of $\mathrm{Si}$ in $\mathrm{W}$ was reached and the filament silicidation process involved the formation and growth of a $\mathrm{W}_{5} \mathrm{Si}_{3}$ outer corona, which for large exposure times was replaced by a Si rich $\mathrm{WSi}_{2}$ outer corona (see Fig. $5 \mathrm{~d}$ and 5e), growing towards the filament core and substituting the pure $\mathrm{W}$ bulk.

The precipitation of the $\mathrm{W}_{5} \mathrm{Si}_{3}$ phase seemed to initiate at a random time since there were samples that showed a larger fraction of $\mathrm{W}$ atoms reacted into $\mathrm{W}_{5} \mathrm{Si}_{3}$ in comparison with other samples obtained from tests with larger exposure times (compare Fig. $4 \mathrm{a}$ and $4 \mathrm{~b}$ ). The randomness of the initiation time of the $\mathrm{W}_{5} \mathrm{Si}_{3}$ precipitation is reflected in Fig. 2 since the aforementioned linear regime of $R_{\mathrm{fil}}(t)$, linked to the precipitation of $\mathrm{W}_{5} \mathrm{Si}_{3}$, was reached at different times for each test.

It can also be seen that the precipitation of silicides was not homogeneous all over the filament surface (see Fig. $4 \mathrm{~b}$ where the nucleation of $\mathrm{W}_{5} \mathrm{Si}_{3}$ is initiating at a localized point of the filament cross section). This non-homogeneity is confirmed if one calculates the portion of $\mathrm{W}$ atoms reacted into $\mathrm{W}_{5} \mathrm{Si}_{3}$ in samples taken from different locations of the central portion of the same tested filament (this is the case of Fig. $4 \mathrm{c}$ 
and Fig. 4d). This suggests that the silicides nucleation process strongly depends on the enhanced at surface defect sites like grain boundaries, dislocation clusters or microcracks, where the superficial energy is lower $[22,23]$. However, once the silicides precipitate locally at the surface of the filament, the local emissivity increases. This produces a non-local decreasing of the filament temperature, the extension of which depends on the thermal conductivity of the material. This mechanism promotes the lateral diffusion of silicides precipitation, which is expected to be much faster than bulk diffusion. In our case, it seems that the nucleation of silicides was extended all over the filament surface in a few minutes.

Regarding the filaments cold end regions, it was observed for all cases that a $\mathrm{WSi}_{2}$ front (composition measured by means of EDX analysis) was rapidly formed during the first minutes of the deposition process. After a first period of around 20 minutes, these $\mathrm{WSi}_{2}$ fronts started propagating from the cold ends towards the middle of the filament at a constant velocity $v=4.0 \pm 0.3 \cdot 10^{-4} \mathrm{~m} / \mathrm{min}$.

\subsection{Influence of the pre-deposition annealing of the filaments in the silicidation} nucleation process

As-manufactured $\mathrm{W}$ filaments may have a large density of structural defects inhomogeneously distributed along the filament length. Such inhomogeneities may come from the extrusion process and the grain size distribution of the metal powder used to fabricate the filaments. When metals are subject to high temperature annealing treatments, the metal crystal grains tend to increase in size and aggregate, resulting in a more homogenously distributed and lower density of structural defects (such as grain boundaries or dislocation clusters) [23]. Even micro-fractures or striations caused during the extrusion of the filament in its manufacturing process can be reduced by the self-diffusion of W atoms over the filament surface [8]. As a result, when the tested filaments were subject to an annealing pre-treatment, the silicides nucleation process and the further growth of the formed silicide layers became much less random. This entailed a repetitive trend in the corresponding $R_{\mathrm{fil}}(t)$ curves as shown by the three black curves of Fig. 6 corresponding to three different filaments subjected to the same annealing pre-treatment. Three different stages can be clearly identified: Firstly, an initial transient period which lasted around $20 \mathrm{~min}$ where $R_{\text {fil }}$ rapidly increased for the first minutes. This initial trend of $R_{\mathrm{fil}}(t)$ is attributed to the formation of the $\mathrm{WSi}_{2}$ silicide fronts at the cold ends of the filament. Secondly, a linear regime with a small slope of $0.010 \pm 0.002 \Omega / \mathrm{min}$ is observed. As explained in sub-section 3.4, this first linear regime of $R_{\mathrm{fil}}(t)$ is reached as a consequence of the $\mathrm{WSi}_{2}$ fronts propagating from the filament cold ends towards the middle of the filament at constant velocity $v$. Finally, an abrupt increase of the slope, up to $0.055 \pm 0.006 \Omega / \mathrm{min}$, led to a second linear regime comparable to those achieved in the $R_{\mathrm{fil}}(t)$ curves obtained from filaments without any pre-deposition annealing treatment (Fig.2). Fig. 6 shows that this second linear regime, linked to the precipitation of $\mathrm{W}_{5} \mathrm{Si}_{3}$, began in all cases at approximately $t=t_{0}=60 \mathrm{~min}$. All three monitored samples (black lines in Fig. 6) did not show any jump in $R_{\mathrm{fil}}(t)$ close 
to $t=t_{0}$. As explained in the next sub-section, this means that also in these cases the $\mathrm{W}_{5} \mathrm{Si}_{3}$ phase started precipitating locally and afterwards, it was rapidly extended over all the filament surface. In fact, if the precipitation of the $\mathrm{W}_{5} \mathrm{Si}_{3}$ started simultaneously over all the central part of the filament, a significant jump as the one showed by the grey curve in Fig. 6 should be visible in the monitored $R_{\text {fil }}(t)$ but clearly, this was not the case.

\subsection{Electric resistance model of the filaments}

Fig. 7 shows schematically the partition of half portion of a silicided filament which has been used to build the electric model that explains the observed $R_{\mathrm{fil}}(t)$ curves shown in Fig. 6. The whole filament can be partitioned into portions with three different characteristics: 1) Two portions of filament, completely transformed into $\mathrm{WSi}_{2}$, comprised between both cold ends and the formed $\mathrm{WSi}_{2}$ fronts expanding towards the middle of the filament at constant velocity $v ; 2$ ) two transition zones which are considered to reach a stationary state once the two $\mathrm{WSi}_{2}$ fronts are formed at the cold ends and that represent a constant and small contribution to $R_{\text {fil }}$, and, finally, 3 ) the central portion of the filament that is progressively transformed into $\mathrm{W}_{5} \mathrm{Si}_{3}$, thus leading to a parallel association of the resistances associated to each material.

To check the validity of the assumptions made regarding the transition zones (portion 2 in Fig 7) an optical microscope image (proportions are not maintained) and a Raman spectroscopy signal mapping of such a transition zone is shown in Fig. 8 for a silicided filament. The areas covered by the $\mathrm{WSi}_{2}$ and $\mathrm{W}_{5} \mathrm{Si}_{3}$ silicide phases have been marked with blue and red colour respectively. The dark spots correspond to pure $\mathrm{W}$ regions of the filament or, alternatively, to regions wherein it was detected a large fluorescent signal from the resin matrix wherein the sample had been embedded. The red square corresponds to the area scanned bymeans of Raman spectroscopy $(5 \mathrm{~mm} \times 160 \mu \mathrm{m})$. The green lines delimit the estimated $\mathrm{W} / \mathrm{W}_{5} \mathrm{Si}_{3}$ and $\mathrm{WSi}_{2} / \mathrm{W}_{5} \mathrm{Si}_{3}$ interfaces. By looking at Fig. 8, one can certify that the length of such transition zones is only of the order of few $\mathrm{mm}$, that is to say, much smaller than $L_{\mathrm{fil}}$. Thus, their contribution to $R_{\mathrm{fil}}$ can be considered practically constant and small enough, having a negligible influence on the following calculation.

The temperature dependence of the emissivity and the electric resistivity $[3,4,12]$ of the involved materials $\left(\mathrm{W}, \mathrm{W}_{5} \mathrm{Si}_{3}, \mathrm{WSi}_{2}\right)$ as well as the volumetric ratio between each material and pure $\mathrm{W}$ were taken into account to calculate $R_{\mathrm{fil}}(t)$. More details regarding this electric model and the following calculations can be found in [18-19].

According to this model, and under our specific deposition conditions, after an initial transient period a first linear regime of $R_{\mathrm{fil}}(t)$ for $t<t_{0}$, i.e. before the precipitation of $\mathrm{W}_{5} \mathrm{Si}_{3}$, should be observed. The slope of this linear regime is given by the following equation:

$$
\mathrm{d} R_{\mathrm{fil}} / \mathrm{d} t \approx 2 v\left(R^{\prime}{ }_{W S i 2}\left(1550^{\circ} \mathrm{C}\right)-R^{\prime}{ }_{W}\left(1900^{\circ} \mathrm{C}\right)\right)
$$


which depends on the propagation velocity $v$ of the $\mathrm{WSi}_{2}$ fronts and where $R^{\prime}{ }_{W S i 2}\left(1550^{\circ} \mathrm{C}\right)$ and $R^{\prime}{ }_{W}\left(1900^{\circ} \mathrm{C}\right)$ are the electric resistances per unit length of a portion of filament exclusively composed by $\mathrm{WSi}_{2}$ and $\mathrm{W}$ respectively, at their specific local temperatures written in brackets. The specific local temperatures of these $\mathrm{W}$ and $\mathrm{WSi}_{2}$ portions of filament have been calculated from the equality between the local power per unit length dissipated by Joule effect and the irradiated power per unit length derived from Stefan-Boltzmann law:

$$
R^{\prime}(T) \mathrm{I}^{2}=e(T) \cdot\left(\beta \pi d_{\mathrm{fil}}\right) \sigma T^{4}
$$

where $T$ is the local temperature given in Kelvin degrees, $I$ is the electric current, $\beta$ expresses the relative expansion of the filament radius due to the conversion of pure $\mathrm{W}$ into its silicided phases (see Table 1), $\sigma$ is the Stefan-Boltzmann constant and where the local electric resistance per unit length $R$ ' and the local emissivity $e$ are temperature dependant. The temperature dependences of the electrical resistivity and the emissivity of $\mathrm{W}, \mathrm{WSi}_{2}$ and $\mathrm{W}_{5} \mathrm{Si}_{3}$ have been extracted from the data given in $[3,4,12,16,17]$ and their values at the specific temperatures considered in our calculations are summarised in Table 1. According to our estimations $R^{\prime}{ }_{W S i 2}\left(1550^{\circ} \mathrm{C}\right)=57 \pm 3 \Omega / \mathrm{m}$ and $R^{\prime}{ }_{W}\left(1900^{\circ} \mathrm{C}\right)=52 \pm 3 \Omega / \mathrm{m}$. Using the measured propagation velocity $v=4.0 \pm 0.3 \cdot 10^{-4}$ $\mathrm{m} / \mathrm{min}$, the maximum value of the slope of $R_{\mathrm{fil}}(t)$ for the linear regime stated by Eq. 1 should be $0.009 \Omega / \mathrm{min}$, which is very close to the measured experimental value, i.e. $0.010 \pm 0,002 \Omega / \mathrm{min}$.

Close to $t=t_{0}$ the $\mathrm{W}_{5} \mathrm{Si}_{3}$ phase starts precipitating into the central portion of the filament. As mentioned in the previous sub-section, if the nucleation of $\mathrm{W}_{5} \mathrm{Si}_{3}$ were homogeneous all over the filament surface, an abrupt decrease of $R_{\text {fil }}$ would be expected at $t=t_{0}$. In fact, due to the considerably higher emissivity of $\mathrm{W}_{5} \mathrm{Si}_{3}$ in comparison to that of pure $\mathrm{W}$, the first consequence should be an abrupt decrease of the temperature of the central part which, in fact, would continue to be composed practically exclusively of tungsten. Thus, it should be observed a jump of

$$
\Delta R_{\mathrm{fil}}\left(\mathrm{t}=\mathrm{t}_{0}\right)=\left(R^{\prime}{ }_{W}\left(1750^{\circ} \mathrm{C}\right)-R^{\prime}{ }_{W}\left(1900^{\circ} \mathrm{C}\right)\right) \cdot\left(L_{\mathrm{fil}}-2 v t_{0}\right) \approx 1 \Omega
$$

where here, the electric resistance per unit length of a pure $\mathrm{W}$ portion of filament at $1750{ }^{\circ} \mathrm{C}$ was estimated to be $R{ }^{\prime}{ }_{W}\left(1750^{\circ} \mathrm{C}\right)=48 \pm 3 \Omega / \mathrm{m}$ following the same procedure as above. This fact was not observed in any of the performed experiments thus meaning that the nucleation of $\mathrm{W}_{5} \mathrm{Si}_{3}$ was not homogeneous.

In the case of annealed pre-treated filaments, it is not clear whether the $\mathrm{W}_{5} \mathrm{Si}_{3}$ phase is propagating into the central portion of the filament starting from the transition zones, where this phase is already present, or if it directly starts nucleating locally on the central part of the filament. Nevertheless, it seems that once the nucleation of $\mathrm{W}_{5} \mathrm{Si}_{3}$ began, lateral diffusion extended the silicide precipitation all over the surface of the central part of the filament in a few minutes. Given this, considering a homogenous thickness for the $\mathrm{W}_{5} \mathrm{Si}_{3}$ outer corona along the central portion of the filament seems to be a reasonable assumption for $t \geq t_{0}$. If we restrict our calculation to a first order 
approximation for the evolution of $R_{\mathrm{fil}}(t)$ with time and we assume that the thickness of the $\mathrm{W}_{5} \mathrm{Si}_{3}$ outer corona was homogeneous over all the central portion of the filament, a second linear regime characterized by a slope given by

$$
\begin{aligned}
& \mathrm{d} R_{\mathrm{fil}} / \mathrm{d} t \approx 2 v\left(R^{\prime}{ }_{W S i 2}\left(1550^{\circ} \mathrm{C}\right)-R^{\prime}{ }_{W}\left(1750^{\circ} \mathrm{C}\right)\right)+\left(\left(R^{\prime}{ }_{W S S i 3}\left(1750^{\circ} \mathrm{C}\right)-\right.\right. \\
& \left.\left.R^{\prime}{ }_{W}\left(1750^{\circ} \mathrm{C}\right)\right) / t_{C}\right) \cdot\left(L_{\mathrm{fil}}-2 v t_{0}\right)
\end{aligned}
$$

should be observed for $t>t_{0}$. Here, $R^{\prime}{ }_{W 5 S i 3}\left(1750^{\circ} \mathrm{C}\right)$ is the electric resistance per unit length of a filament portion that is exclusively composed by $\mathrm{W}_{5} \mathrm{Si}_{3}$ at a temperature of $1750^{\circ} \mathrm{C}$ and it was estimated to be $R^{\prime}{ }_{W 5 S i 3}\left(1750^{\circ} \mathrm{C}\right)=64 \pm 3 \Omega / \mathrm{m}$ while $t_{\mathrm{c}}$ is the amount of time needed by the $\mathrm{W}_{5} \mathrm{Si}_{3}$ outer layer to reach the filament core at the central portion of the filament. From the data obtained from Figs. 4 and $5, t_{\mathrm{c}}$ can be estimated to be of the order of $100 \mathrm{~min}$. We must notice that during this period of time $\left(t_{o} t_{c}\right)$, the temperature of the central part of the filament $\left(\sim 1750^{\circ} \mathrm{C}\right)$ does not change significantly because the effect of the increment of the electrical resistivity is compensated by the increment of the filament radius in said portion of filament. According to the presented model, the slope of $R_{\mathrm{fil}}(t)$ for this second linear regime should be included between $0.055 \pm 0.015 \Omega$ $/ \mathrm{min}$, which clearly is the case. These results confirm that the linear regimes of $R_{\mathrm{fil}}(t)$ observed in Fig. 2 are effectively linked to the precipitation of $\mathrm{W}_{5} \mathrm{Si}_{3}$.

As a remark, we may refer now to the inset of Fig. 2, wherein the complete $R_{\mathrm{fil}}(t)$ evolution of a silicidation test that lasted 190 min is shown. The corresponding samples, collected from the central portion of the tested filament, showed a large presence of the $\mathrm{WSi}_{2}$ phase. The dramatic decay of $R_{\mathrm{fil}}(t)$, subsequent to the second linear regime, is therefore assumed to be directly linked to the precipitation of $\mathrm{WSi}_{2}$. Such a decay of the electric resistance is given by a large drop of the local temperature at the central portion of the filament. The drop of the local temperature is a consequence of the larger emissivity of $\mathrm{WSi}_{2}$ and the increase in the filament volume (given by the volumetric ratio between $\mathrm{WSi}_{2}$ and $\mathrm{W}_{5} \mathrm{Si}_{3}$ or $\mathrm{W}$ ).

\subsection{Influence of the $\mathrm{H}_{2}$ dilution on the silicides nucleation process}

Fig. 9 shows the $R_{\mathrm{fil}}(t)$ curve of a silicidation test corresponding to the third series. For such deposition conditions, we found that $t_{0} \approx 120 \mathrm{~min}$, which is a two fold increase with respect to the value of $t_{0}$ found in those tests wherein only pure $\mathrm{SiH}_{4}$ was used as precursor gas. Such effect could be explained by the Si etching properties [24] of the atomic hydrogen generated at the filament surface by the catalytic dissociation of $\mathrm{H}_{2}$ molecules. However, the slopes of $R_{\mathrm{fil}}(t)$ for $t<t_{0}$ and $t>t_{0}$ (around $0.01 \Omega / \mathrm{min}$ and 0.06 $\Omega /$ min respectively) are practically the same than those obtained from the tests without any $\mathrm{H}_{2}$ dilution. This suggests that hydrogen dilution has a remarkable influence on the nucleation of silicides in the central part of the filament, which is delayed in time, but not on the growth rate of the corresponding precipitates.

\section{Conclusions}


According to our study, the silicidation process of $\mathrm{W}$ filaments at high temperature formation of two $\mathrm{WSi}_{2}$ fronts at the cold ends. After an initial transient period, these $\mathrm{WSi}_{2}$ fronts start propagating towards the middle of the filament at constant velocity. Regarding the central portion of the filament, three different stages have been identified: the stage of $\mathrm{Si}$ atoms dissolution in the $\mathrm{W}$ bulk; the precipitation and further growth of a $\mathrm{W}_{5} \mathrm{Si}_{3}$ outer corona, and its subsequent transformation into the $\mathrm{Si}$-richer $\mathrm{WSi}_{2}$ silicide phase.

An electric model has been exposed to correlate the different stages observed in the monitored $R_{\mathrm{fil}}(t)$ curves with the different stages of the filaments silicidation process elucidated from the SEM, EDX and Raman analyses of the collected samples. In this way, real time information about the silicidation process affecting the filaments was obtained by monitoring their electric resistance. We have shown that each of these silicidation stages leaves a characteristic trace on the filaments electric resistance time evolution $R_{\mathrm{fil}}(t)$.

Regarding the central portion of the filament, it has been shown that the duration of the $\mathrm{Si}$ atoms dissolution stage, which determines the moment at which $\mathrm{W}_{5} \mathrm{Si}_{3}$ precipitation will occur, is highly influenced by the initial superficial status of the filaments. Thus, the precipitation of $\mathrm{W}_{5} \mathrm{Si}_{3}$ may begin from the very start of the deposition process. However, if the filaments are subject to an annealing pre-treatment, the duration of this first stage is stabilised around $60 \mathrm{~min}$. This leads to a clearly repetitive trend in the monitored $R_{\mathrm{fil}}(t)$ curves. This last effect is attributed to a reduction and a homogenisation of the initial defects distribution at the filaments surface. It has also been found that the addition of molecular hydrogen to the silane atmosphere influences the nucleation process of the $\mathrm{W}_{5} \mathrm{Si}_{3}$ silicide phase, increasing the duration of the $\mathrm{Si}$ atoms dissolution stage, but does not have any influence on the growth process of the formed $\mathrm{W}_{5} \mathrm{Si}_{3}$ layer.

Finally, the use of the electrical model to interpret the observed trends of $R_{\mathrm{fil}}(t)$ made also possible to show that the precipitation of $\mathrm{W}_{5} \mathrm{Si}_{3}$ always nucleates locally. However, once the nucleation of $\mathrm{W}_{5} \mathrm{Si}_{3}$ starts, lateral diffusion extends the silicide precipitation all over the surface of the central part of the filament in a few minutes.

\section{Acknowledgements}

This work has been supported by the Ministerio de Ciencia e Innovación, the Ministerio de Economía y Competitividad and the European Regional Development Fund through the projects AMIC (ENE2010-21384-C04-03), MICROSIL08 (PSE-120000-2008-1) and INNDISOL (IPT-420000-2010-6).

\section{References}


[1] D. Landsperger (Ed.), Outlook for tungsten production and applications in the 21st century, 9th Int. Tungsten Symposium, International tungsten industry association, Pittsburgh, USA, 2002.

[2] L. Bartha, in: C.L. Briant, J.J. Petrovic, B.P. Bewlay, A.K. Vasudevan, H.A. Lipsitt (Eds.), High Temperature Silicides and Refractory Alloys, vol. 322, MRS Symp. Proc., Pittsburgh, PA, 1993, pp. 531-536.

[3] C. Gelain, A. Cassuto, and P. Le Goff, Oxid. Met. 3 (1971) 115-138.

[4] F. Nava, K.N. Tu, E. Mazzega, M. Michelini, and G. Queirolo, J. Appl. Phys. 61 (1987) 1085-1093.

[5] A.I. Barg, B.S. Bokstein, L.M. Klinger, J. Appl. Phys. 72 (1992) 1356-61.

[6] K.H. Lee, J.K.Yoon, J.K. Lee, J.M. Doh, K.T. Hong, W.Y. Yoon, Surf. Coat. Technol. 187 (2004) 146-153.

[7] J.K. Yoon, K.W. Lee, S.J. Chung, I.J. Shon, J.M. Doh, G.H. Kim, J. Alloys Compd. 420 (2006) 199-206.

[8] J. K. Holt, M. Swiatek, D. G. Goddwin, H. A. Atwater, J. Appl. Phys. 92 (2002) 4803-4808.

[9] E. Iwaniczko, A.H. Mahan, B. Yan, L.N. Gedvilas, D.L. Williamson, P. Nelson in: J.R. Abelson, G. Ganguly, H. Matsumura, J. Robertson, E. Schiff (Eds.), MRS Symp. Proc. 762 , A7.8 (2003).

[10] N. Kniffler, A. Pueger, D. Scheller, B. Schroeder, Thin Solid Films 517 (2009), 3424-3426.

[11] P.A.T.T. van Veenendaal, O.L.J. Gijzeman, J.K. Rath, R.E.I. Schropp, Thin Solid Films 395(2001) 194-197.

[12] K. Honda, K. Ohdaira, H. Matsumura, Jpn. J. Appl. Phys. 47 (2008) 3692-3698.

[13] C.H.M. van der Werf, P.A.T.T. van Veenendaal, M.K. van Veen, A.J. Hardeman, M.Y.S. Rusche, J.K. Rath, R.E.I. Schropp, Thin Solid Films 430 (2003) 46-49.

[14] O. Nos, P.A. Frigeri, J. Bertomeu, Thin Solid Films 519 (2011) 4531-4534.

[15] P.A. Frigeri, O. Nos, J.D. Calvo, P. Carreras, R. Roldan, J.M. Asensi, J. Bertomeu, Phys. StatusSolidi C, 7 (2010) 588-591.

[16] P. D. Desai, T.K. Chu, M. James, C. Y. Ho, Phys. Chem. Ref. Data, 13 (1984) 1069.

[17] E. Lassner, W. D. Schubert, Tungsten, Kluwer Academic. Plenum Publishers, New York, 1999.[18] P.A. Frigeri, O. Nos, J. Bertomeu, 7th International Conference on Hot-WireChemicalVaporDepositionProceedings, October 8-12 (2012), Osaka, Japan.

[19] O. Nos, HWCVD technology development addressed to the high rate deposition of $\mu c-S i: H$, PhD Thesis, Universitat de Barcelona, Spain (2013). 
[20] A. Laukart, T. Harig, M. Höfer, L. Schäfer, 7th International Conference on HotWire Chemical Vapor Deposition Proceedings, October 8-12 (2012), Osaka, Japan.

[21] W. Pletschen, N. Herres, M. Maier, M. Seelmanneggebert, J. Wagner, A. Voigt, H.P. Strunk, Appl. Surf. Sci. 38 (1989) 259-268.

[22] R.E. Reed-Hill, Physical Metallurgy Principles, third ed., PWS Publishing Company, Boston, 1994.

[23] J.D.Verhoeven, Fundamentals of Physical Metallurgy, first ed.,John Wiley and Sons, New York, 1975.

[24] K. Uchida, A. Izumi, H. Matsumura, Thin Solid Films 395(2001) 75-77. 
Table 1

\begin{tabular}{l|cccc} 
& $\rho[\Omega \cdot \mathrm{cm}]$ & $e$ & $\beta$ & $\mathrm{T}\left[{ }^{\circ} \mathrm{C}\right]$ \\
\hline $\mathrm{W}$ & $63 \pm 4$ & $0.27 \pm 0.015$ & 1 & 1900 \\
$\mathrm{~W}$ & $58 \pm 4$ & - & 1 & 1750 \\
$\mathrm{~W}_{5} \mathrm{Si}_{3}$ & $135 \pm 6$ & $0.35 \pm 0.015$ & 1.31 & 1750 \\
$\mathrm{WSi}_{2}$ & $188 \pm 10$ & $0.38 \pm 0.015$ & 1.64 & 1550
\end{tabular}




\section{Table Captions}

Table 1. Resistivity and emissivity of $\mathrm{W}, \mathrm{WSi}_{2}$ and $\mathrm{W}_{5} \mathrm{Si}_{3}$ at the different temperatures of interest. Factor $\beta$ has been calculated from the volumetric ratio between each material and pure tungsten. 


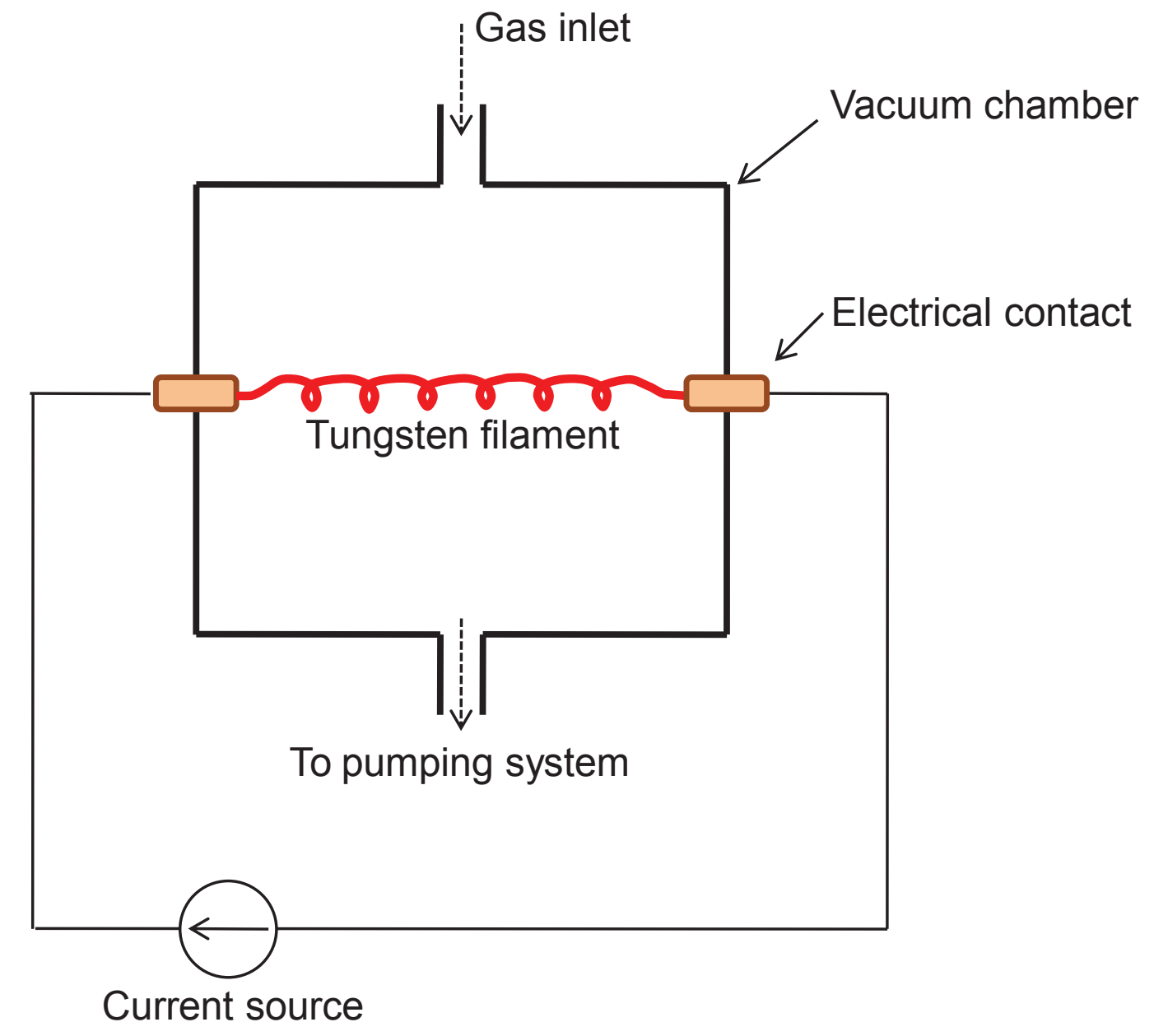




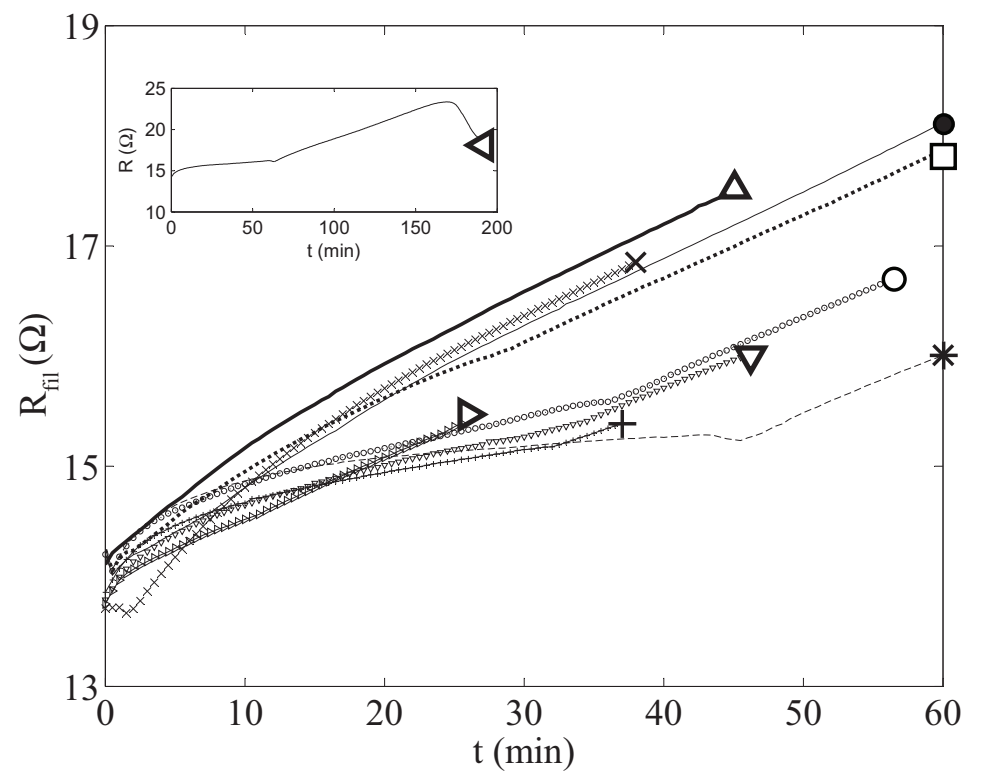




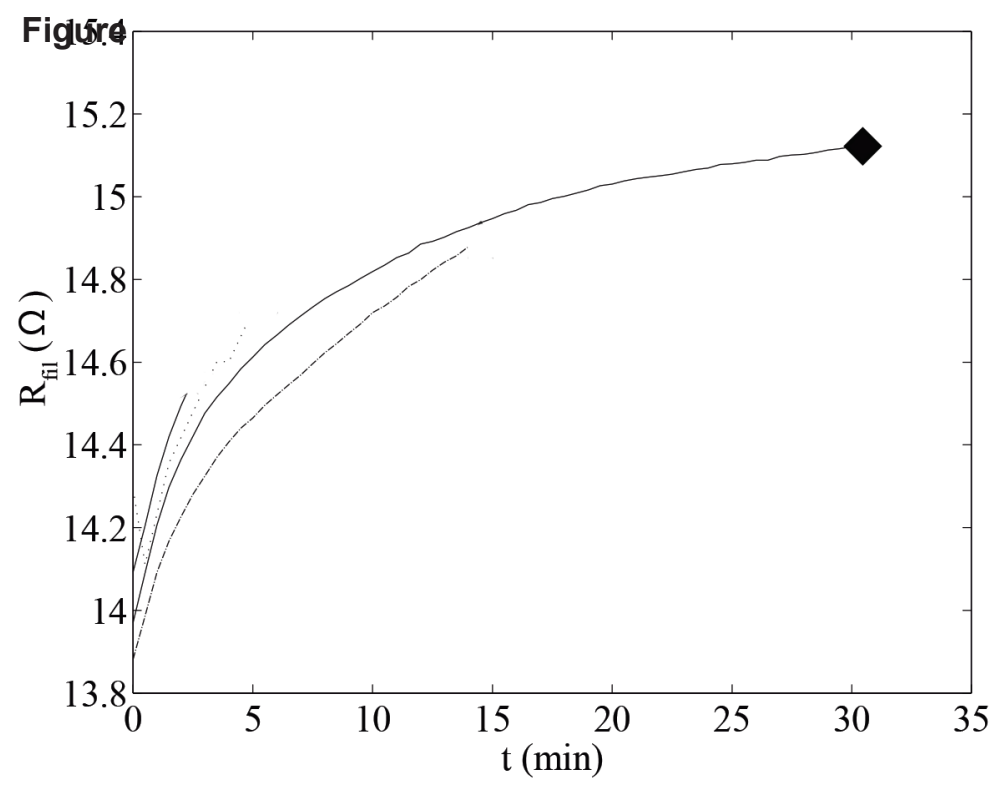



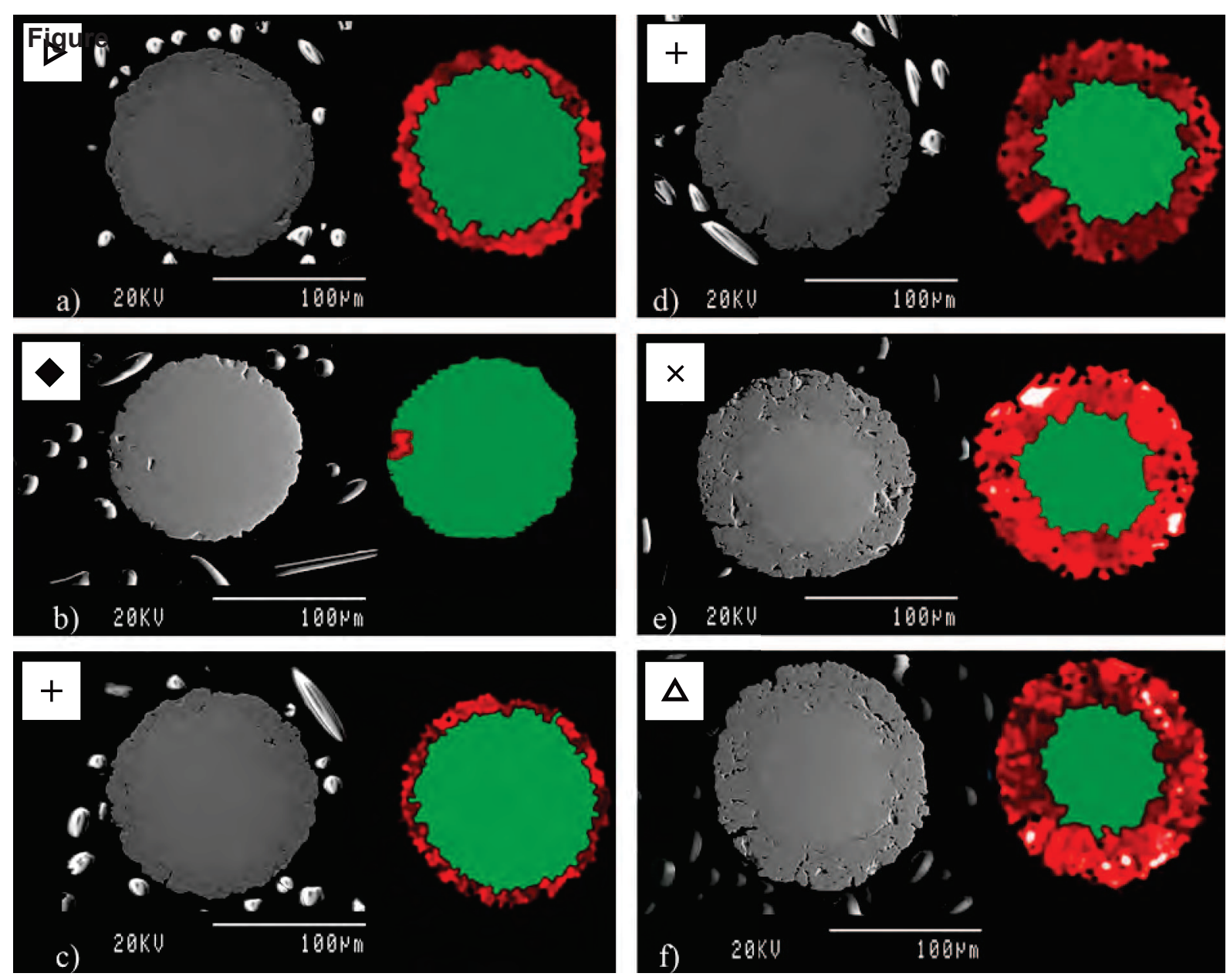

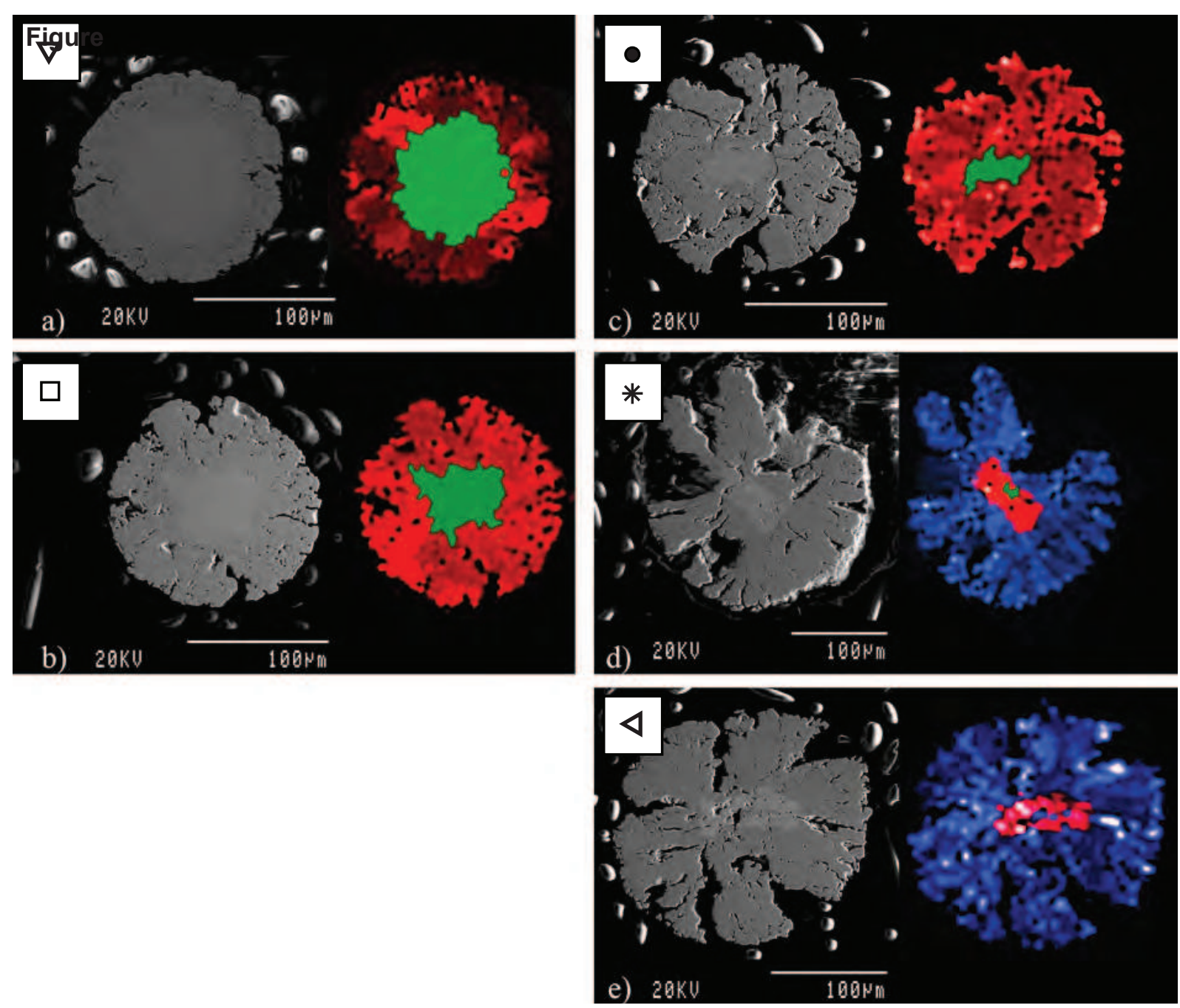


\section{Figure}

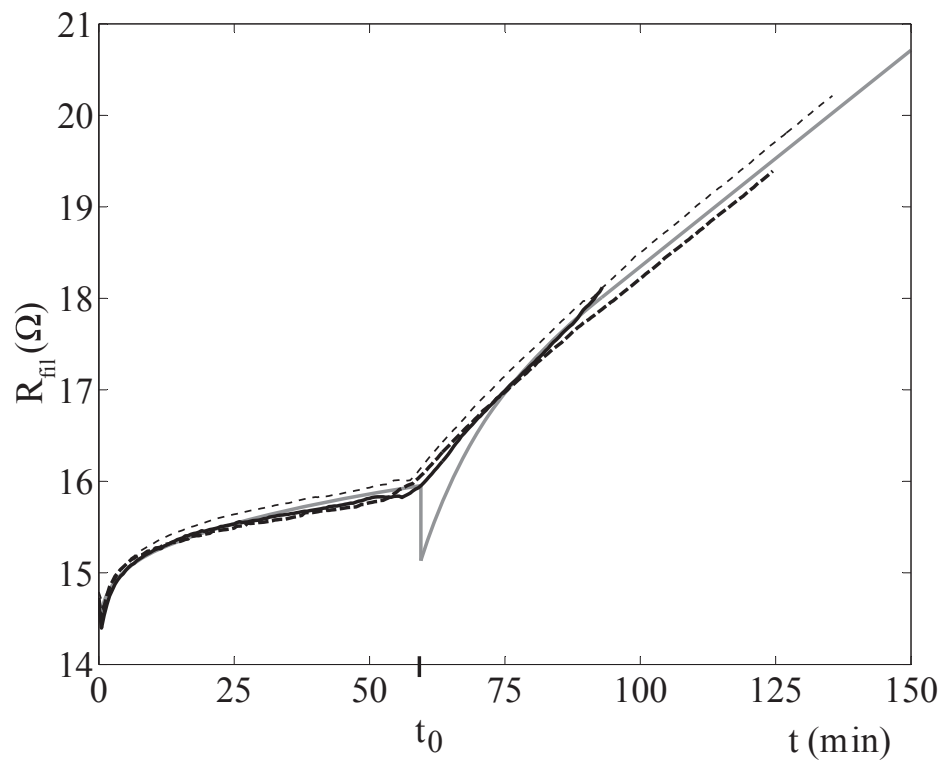


Figure

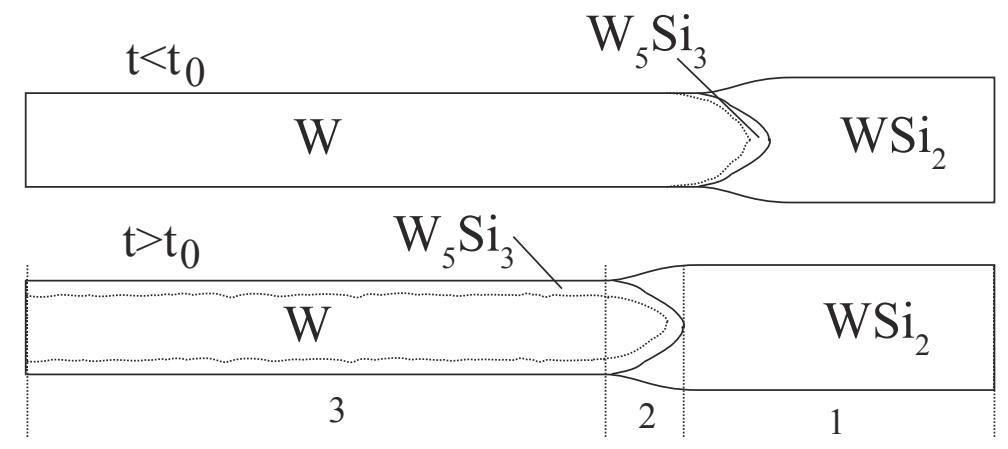



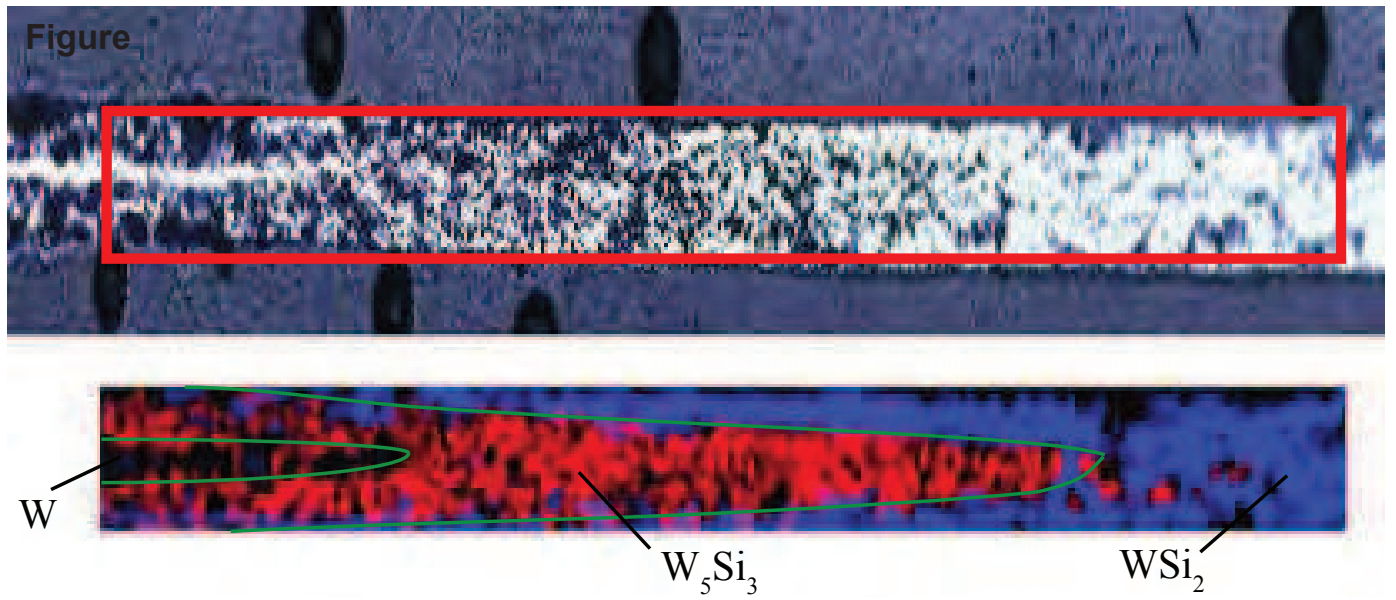


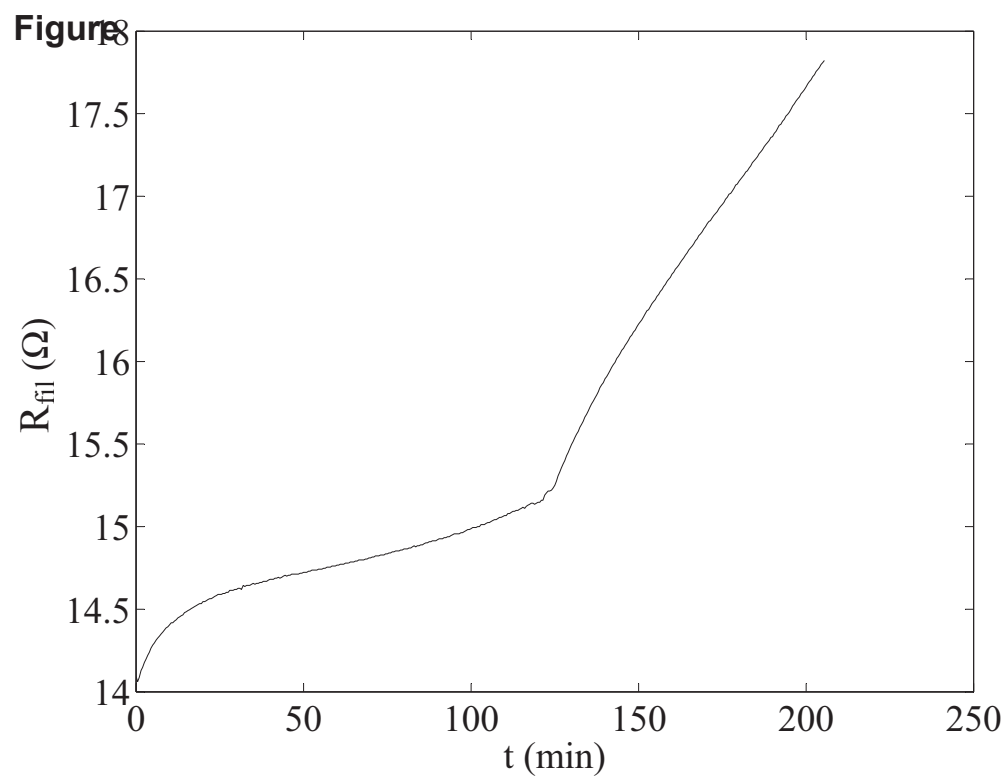




\section{Figure captions}

Fig. 1 Schematic overview of the experimental setup used for the filament ageing study.

Fig. $2 R_{\mathrm{fil}}(t)$ curves of the silicidation tests $\left(d_{\mathrm{fil}}=0.125 \mathrm{~mm}, I=1.63 \mathrm{~A}, p=2 \mathrm{~Pa}, \Phi_{\mathrm{SiH} 4}=12\right.$ sccm) performed with $\mathrm{W}$ filaments that resulted in samples with silicides formation. In particular, the samples $\bullet, \square, *$ and $\triangleleft$ (see inset) correspond to silicidation tests with a total silane exposure time of $90 \mathrm{~min}, 75 \mathrm{~min}, 140 \mathrm{~min}$ and $190 \mathrm{~min}$ respectively.

Fig. $3 R_{\mathrm{fil}}(t)$ curves of the silicidation tests $\left(d_{\mathrm{fil}}=0.125 \mathrm{~mm}, I=1.63 \mathrm{~A}, p=2 \mathrm{~Pa}, \Phi_{\mathrm{SiH} 4}=12\right.$ sccm) performed with $\mathrm{W}$ filaments that resulted in samples where silicides formation is absent.

Fig. 4 Cross sectional SEM images (left) and the corresponding Raman signal mappings (right) of silicided W filaments $\left(d_{\mathrm{fil}}=0.125 \mathrm{~mm}, I=1.63 \mathrm{~A}, p=2 \mathrm{~Pa}, \Phi_{\mathrm{SiH} 4}=12 \mathrm{sccm}\right)$.

The accumulated $\mathrm{SiH}_{4}$ exposure times are: $26 \mathrm{~min}(\mathrm{a}), 30 \mathrm{~min}$ (b), $37 \mathrm{~min}$ (c,d), $38 \mathrm{~min}$

(e) and $45 \mathrm{~min}$ (f). Green and red colours in the Raman signal mappings indicate the presence of pure $\mathrm{W}$ and $\mathrm{W}_{5} \mathrm{Si}_{3}$ respectively. Used symbols correspond to those represented in Figs. 2 and 3.

Fig. 5 Cross sectional SEM images (left) and the corresponding Raman signal mappings (right) of silicided W filaments $\left(d_{\mathrm{fil}}=0.125 \mathrm{~mm}, I=1.63 \mathrm{~A}, p=2 \mathrm{~Pa}, \Phi_{\mathrm{SiH} 4}=12 \mathrm{sccm}\right.$ ).

The accumulated $\mathrm{SiH}_{4}$ exposure times are: $46 \mathrm{~min}$ (a), $75 \mathrm{~min}$ (b), $90 \mathrm{~min}$ (c), 140min (d) and 190 min (e). Green and red colours in the Raman signal mappings indicate the presence of pure $\mathrm{W}$ and $\mathrm{W}_{5} \mathrm{Si}_{3}$ respectively. Used symbols correspond to those represented in Fig. 2.

Fig. 6 Black lines correspond to $R_{\mathrm{fil}}(t)$ curves of three silicidation tests $\left(d_{\mathrm{fil}}=0.125 \mathrm{~mm}\right.$, $\left.I=1.63 \mathrm{~A}, p=2 \mathrm{~Pa}, \Phi_{\mathrm{SiH} 4}=12 \mathrm{sccm}\right)$ performed with $\mathrm{W}$ filaments that were subject to a pre-deposition annealing treatment $\left(30 \mathrm{~min}\right.$ in vacuum at $\left.1900^{\circ} \mathrm{C}\right)$. The grey line shows the qualitative behaviour of $R_{\mathrm{fil}}(t)$ for a filament if the silicide precipitation at the central part was nucleating homogeneously.

Fig. 7 Schematic view of the different filament regions (1: $\mathrm{WSi}_{2}$ front; 2 : transition region; 3 : central portion of the filament) considered by the proposed electric model during its silicidation process; before (top) and after (bottom) the precipitation of silicides at the central portion of filament.

Fig. 8 Longitudinal cross section optical image (top) and the corresponding Raman signal mapping (bottom) of a silicided W filament. Red and blue colours in the Raman signal mapping indicate the presence of $\mathrm{W}_{5} \mathrm{Si}_{3}$ and $\mathrm{WSi}_{2}$ respectively. The red square corresponds to an area of $5 \mathrm{~mm} \times 160 \mu \mathrm{m}$ (proportions are not maintained). The green lines correspond to the estimated $\mathrm{W} / \mathrm{W}_{5} \mathrm{Si}_{3}$ and $\mathrm{WSi}_{2} / \mathrm{W}_{5} \mathrm{Si}_{3}$ interfaces.

Fig. $9 R_{\text {fil }}(t)$ curve of a silicidation test performed with a W filament using $\mathrm{SiH}_{4}$ and $\mathrm{H}_{2}$ as precursor gas mixture $\left(d_{\mathrm{fil}}=0.125 \mathrm{~mm}, I=1.63 \mathrm{~A}, p=10 \mathrm{~Pa}, \Phi_{\mathrm{SiH} 4}=12 \mathrm{sccm}\right.$, $\left.\Phi_{\mathrm{SiH} 4}=108 \mathrm{sccm}\right)$. 